\title{
Half-dose, long-acting gonadotropin-releasing hormone agonist (Diphereline) is comparable with daily injections of short-acting gonadotropin-releasing hormone agonist (Suprefact) in IVF/ICSI cycles
}

\author{
Ensieh Sh. Tehraninejad ${ }^{1,2}$, Elham Azimi Nekoo², Zahra Ezabadi ${ }^{1}$, Batool H. Rashidi², \\ Elham Amirchaghmaghi ${ }^{1}$, Elham Pour Matroud ${ }^{2}$
} 1Endocrinology and Female Infertility Department, Royan Institute for Reproductive
Biomedicine, ACECR, Tehran, Iran
${ }^{2}$ Gynecology and Obstetrics Department, Tehran University of Medical Sciences, Tehran,
Iran

Submitted: 2 November 2009

Accepted: 16 March 2010

Arch Med Sci 2010; 6, 6: 945-949

DOI: 10.5114 /aoms.2010.19306

Copyright (c) 2010 Termedia \& Banach

\section{Abstract}

Introduction: The aim of the study was to compare the efficacy of half-dose, long-acting GnRH analogue (Diphereline) with Suprefact in IVF/ICSI (in vitro fertilization/intracytoplasmic sperm injection) cycles.

Material and methods: In this randomized clinical trial performed in Royan Institute, 126 infertile women who were first time candidates for IVF/ICSI were enrolled. Patients were randomly divided into two groups by using a random number table. In one group, 62 patients received a single half dose, $1.87 \mathrm{mg}$ Diphereline, in mid-luteal phase. In the other group, 64 cases were treated with buserelin from the previous mid-luteal phase. $P$ value less than 0.05 was considered significant.

Results: The mean age of patients in the Diphereline and Suprefact groups was $27.9 \pm 3.6$ and $29.6 \pm 3.5$ years, respectively $(p=0.01)$. In the Diphereline group, the mean number of used gonadotropins was $25.6 \pm 12.1$ ampoules, while in the second group it was $25.9 \pm 8.5$ ampoules. Numbers of retrieved and MII oocytes were significantly higher in the Diphereline group $(12.1 \pm 6.3$ and $9.6 \pm 5.5)$ in comparison to the Suprefact group (9.4 \pm 6.4 and $7.2 \pm 5.1)$. Although the number of developed embryos in the Diphereline group was statistically higher than in the Suprefact group ( $6.1 \pm 3.9$ vs. $4.7 \pm 3.4, p=0.04)$ there was no significant difference in pregnancy rate $(37.1 \%, 95 \% \mathrm{Cl}[26.16-49.54]$ vs. $37.5 \%, 95 \% \mathrm{Cl}[26.67-$ 49.75]).

Conclusions: A half-dose, long-acting GnRH agonist can be successfully used in ovarian stimulation and produces a higher number of MII oocytes and embryos. The pregnancy rates with this method are acceptable.

Key words: diphereline, GnRH agonist, assisted reproductive technology, half dose

\section{Introduction}

Ovulation induction is the most critical and important step in ART cycles. In the past, human gonadotropins have been widely used alone; however, during the past few decades, due to a high prevalence of premature LH surge and its negative effects on oocytes [1], gonadotropin-releasing

\author{
Corresponding author: \\ Ensieh Sh. Tehraninejad, MD \\ Endocrinology and Female \\ Infertility Department \\ Royan Institute for Reproductive \\ Biomedicine, ACECR \\ P.O.Box:19395-4644 \\ No 12, East Hafez Street \\ Bani Hashem Street, Resalat \\ Highway \\ Tehran, Iran \\ Phone: +98 2122306480 \\ Fax: +98 2122306481 \\ E-mail: \\ tehraninejad@royaninstitute.org
}


hormone $(\mathrm{GnRH})$ analogues ( $\mathrm{GnRHa}$ ) have been widely used for pituitary desensitization during controlled ovarian stimulation (COS). The long protocol with $\mathrm{GnRHa}$ has been generally accepted for pituitary suppression because of decreases in cancellation rates, prevention of premature luteinization and endogenous luteinizing hormone $(\mathrm{LH})$ surges, an increase in multiple follicular growth [2] and potentially better treatment cycle timing [3].

Nowadays, many types of short- and long-acting $\mathrm{GnRH}$ analogues are being used [4]. The use of a single-dose depot long-acting $\mathrm{GnRH}$ agonist in COS cycles, instead of a daily low-dose preparation, would be more convenient for patients because it eliminates multiple injections and facilitates stimulation [5]. It is welcomed, both by patients and physicians, because of the convenience of a single administration [6]. However, when profound pituitary desensitization is induced, there is an increase in the number of used gonadotropin ampoules and the duration of the COS cycle without improving pregnancy rates or other clinical outcomes [7]. Although some researchers such as Genon et al. showed superiority of a short-acting $\mathrm{GnRH}$-agonist (buserelin) over the long-acting agents (Decapeptyl) in achievement of pregnancy and its outcome [8], others did not find any significant differences in clinical pregnancy rate, implantation rate, and early pregnancy loss rate between the two groups and suggested that a single administration of long-acting $\mathrm{GnRHa}$ is a useful alternative for improving patient convenience, with clinical outcomes comparable to daily administrations of short-acting $\mathrm{GnRHa}$ in $\mathrm{COH}$ (controlled ovarian hyperstimulation), for IVF-ET cycles [9]. In addition to the controversy in type of $\mathrm{GnRH}$ agonist used (long-acting vs. short-acting), another challenging issue is the dose of long-acting GnRH agonist.

Some studies have compared the outcome of halving the dose of $\mathrm{GnRH}$ agonist depot with its full-dose administration [6, 10, 11]. For example, Tsai et al. [12] and Hsieh et al. [13] showed that the halfdose depot leuprolide acetate resulted in comparable clinical outcomes with the standard daily injections in the long GnRHa protocol.

In spite of some studies in this regard, use of a long-acting instead of short-acting form of $\mathrm{GnRH}$ agonist and its dose (full dose or half dose) remain controversial topics in ART.

The aim of this study was to use a single halfdose depot GnRH agonist (Diphereline) instead of the routine daily subcutaneous short-acting form (Suprefact) in the long protocol ICSI/IVF cycles in a randomized clinical trial and compare the stimulation time, number of retrieved oocytes and embryos and pregnancy rate between the two groups.

\section{Material and methods}

In this randomized clinical trial, 126 patients who were first time candidates for ICSI/IVF at Royan Institute were enrolled between April 2005 and March 2006. All patients signed an informed consent form. This study was approved by the Royan Institute Ethics Committee.

The patients were randomly divided into two groups by using a random number table. Because of the different drug administration routes, we could not blind this study. All patients were 20-35 years old. We excluded patients aged more than 35 years old or with follicle-stimulating hormone $(\mathrm{FSH})>8 \mathrm{mIU} / \mathrm{ml}$ because we wanted to survey the efficacy of these two drugs in normoresponsive patients. Except for moderate to severe endometriosis, other causes of infertility were included in this study. In all patients, the serum FSH level was less than $8 \mathrm{mIU} / \mathrm{ml}$ on day 3 of the previous menstrual cycle. Patients with ovarian or pelvic surgery were also excluded.

After randomization, 62 patients in group I (halfdose Diphereline) and 64 patients in group II (daily injection of Suprefact) were studied. All patients underwent transvaginal sonography on the 3rd day of the menstruation cycle; and serum LH, FSH and oestradiol were checked on the same day. Low-dose oral contraceptive pills (ethinyl oestradiol $30 \mu \mathrm{g}$ + levonorgestrel $150 \mu \mathrm{g}$ ) were administered from day 5 of the menstrual period until day 21. On day 21 of the cycle, in group I, a half dose $(1.85 \mathrm{mg})$ of longacting GnRH agonist (Diphereline S.R., 3.75 mg, IPSEN, France) was intramuscularly injected in a single dose, whereas in group II, a subcutaneous GnRH agonist (Suprefact, Hoechst, Germany), at a standard dose of $0.5 \mathrm{mg}$, was started on day 21 and repeated until the new menstruation period. At this time daily doses of Suprefact were reduced to $0.25 \mathrm{mg}$ until human chorionic gonadotropin (hCG) injection. For both groups, from day 3 of the new menstrual cycle, 2 ampoules per day of human menopausal gonadotropin (hMG; Monogon $75 \mathrm{U} \mathrm{FSH/75} \mathrm{U} \mathrm{LH,}$ Ferring, Germany) were intramuscularly injected and at day 6 of gonadotropin stimulation, the first control transvaginal sonography was done. If the follicular response was not adequate, hMG ampoules were increased and serial sonography was performed every other day and repeated until at least 2-3 follicles with 18-20 mm diameter were seen. At that time patients received 10,000 IU hCG (Profasi, 10000 IU, Serono, the Netherlands) injected intramuscularly, and transvaginal oocyte retrieval was performed 3436 hours later. After doing an IVF or ICSI procedure in the laboratory, the embryos were transferred 2-3 days later. The quality of embryos was assessed according to the number of blastomeres and cytoplasmic fragmentation. High quality embryos were defined by $0 \%$ fragmentation and all blastomeres with similar size [14]. 
In both groups on day 8 , if less than 3 follicles with diameters more than $14 \mathrm{~mm}$ were seen, the cycle was cancelled. The number of hMG ampoules and days for ovarian simulation, rate of occurrence of ovarian hyperstimulation syndrome (OHSS), cycle cancellation, number and quality of retrieved oocytes, number of metaphase II oocytes (MII), number and quality of embryos, pregnancy and miscarriage rates were compared between the two groups.

For luteal phase support, all patients received $400 \mathrm{mg}$ vaginal supposition of progesterone (Cyclogest $400 \mathrm{mg}$, Alpharma, England) twice per day starting on the day of oocyte retrieval. Serum $\beta$ hCG levels more than $50 \mathrm{lU} / \mathrm{I}$ were considered as chemical pregnancy. Four to five weeks after embryo transfer, pregnancy results were assessed with TVS.

The Statistical Package for the Social Sciences version 15 software was used to analyse the data. A level of 0.05 was considered significant. Independent samples t-test was used for continuous response measures in both groups' variables. Prevalence differences were analysed by $t$-test, $\chi^{2}$ test and Fisher's exact test.

\section{Results}

In this clinical trial, after randomization, 62 patients in group I (half-dose Diphereline) and 64 patients in group II (daily injection of Suprefact) were studied. About $90 \%$ of studied patients underwent ICSI (90.3\% in group I and $90.6 \%$ in group II). Mean body mass index (BMI) and duration of infertility in the two groups were not significantly different (Table I). Also, the causes of infertility between the two groups were matched (Table I). The difference in mean age between the two groups was statistically significant $(p=0.01)$. There were significant differences in the numbers of retrieved oocytes, metaphase II oocytes and numbers of embryos between the two groups. The mean number of used gonadotropin ampoules, duration of ovarian stimulation, and numbers of good quality embryos and transferred embryos were not significantly different between the groups (Table II).

Table I. Basic characteristics of studied patients

\begin{tabular}{|c|c|c|c|}
\hline & $\begin{array}{l}\text { Group I (Diphereline) } \\
\qquad N=62\end{array}$ & $\begin{array}{c}\text { Group II (Suprefact) } \\
\qquad N=64\end{array}$ & $p$ value \\
\hline Mean age [years] & $27.9 \pm 3.6^{a}$ & $29.6 \pm 3.5^{a}$ & $0.01^{*}$ \\
\hline Mean BMI $\left[\mathrm{kg} / \mathrm{m}^{2}\right]$ & $24.7 \pm 2.5^{a}$ & $25.57 \pm 3.1^{\mathrm{a}}$ & NS \\
\hline Duration of infertility [years] & $7.4 \pm 4.01^{a}$ & $8.6 \pm 3.62^{a}$ & NS \\
\hline Causes of infertility, n (\%) & & & NS \\
\hline Male factor & $32(51.61)^{b}$ & $35(54.68)^{b}$ & \\
\hline Female factor & $20(32.29)^{b}$ & $18(28.12)^{b}$ & \\
\hline PCOs & $7(11.3)^{b}$ & $5(7.8)^{\mathrm{b}}$ & \\
\hline Endometriosis & $4(6.4)^{b}$ & $7(10.9)^{b}$ & \\
\hline Tubal factor & $9(14.5)^{b}$ & $6(9.5)^{b}$ & \\
\hline Unexplained & $10(16.1)^{b}$ & $11(17.2)^{b}$ & \\
\hline
\end{tabular}

apresented as mean $\pm S D$

bpresented as number (percentage)

*Significant difference $(p<0.05)$

NS - not significant

Table II. Early results and clinical outcomes of ART cycles in two groups

\begin{tabular}{|c|c|c|c|}
\hline & $\begin{array}{c}\text { Group I (Diphereline) } \\
\qquad N=62\end{array}$ & $\begin{array}{c}\text { Group II (Suprefact) } \\
\qquad N=64\end{array}$ & $p$ value \\
\hline Ampoules of HMG & $25.6 \pm 12.1^{a}$ & $25.9 \pm 8.5^{a}$ & NS \\
\hline Days of stimulation & $10.32 \pm 2.03^{a}$ & $9.95 \pm 0.91^{a}$ & NS \\
\hline Retrieved oocytes & $12.11 \pm 6.32^{a}$ & $9.43 \pm 6.4^{a}$ & $0.02^{*}$ \\
\hline Oocytes in stage MII & $9.6 \pm 5.54^{a}$ & $7.23 \pm 5.12^{a}$ & $0.015^{*}$ \\
\hline Numbers of embryos & $6.1 \pm 3.9^{a}$ & $4.73 \pm 3.45^{a}$ & $0.04^{*}$ \\
\hline Numbers of transferred embryos & $2.31 \pm 0.733^{a}$ & $2.29 \pm 0.726^{a}$ & NS \\
\hline Numbers of morphologically good embryos & $4.9 \pm 3.2^{a}$ & $3.9 \pm 3.1^{a}$ & NS \\
\hline Clinical pregnancy rate [\%] & 37.1 & 37.5 & NS \\
\hline Miscarriage rate [\%] & 8.7 & 8.33 & NS \\
\hline Number of ICSI cycles, $n(\%)$ & $56(90.3)$ & $58(90.6)$ & NS \\
\hline
\end{tabular}

apresented as mean $\pm S D$

NS - not significant

* Significant difference $(p<0.05)$ 
Pregnancy rate in the Diphereline group was $37.1 \%(95 \% \mathrm{Cl}[26.16-49.54])$ (23 from 62 patients) and $37.5 \%(95 \% \mathrm{Cl}$ [26.67-49.75]) (24 from 64 patients) in the Suprefact group but this difference was not statistically significant $(p=0.92)$. In group I, 17 pregnancies were single, 4 pregnancies were twins, and 2 miscarriages occurred. In group II, 20 pregnancies were single, 2 pregnancies were twins, and 2 miscarriages occurred. These differences between the two groups were not statistically significant. Severe OHSS did not occur in either groups. There was no cancellation of treatment in either group.

\section{Discussion}

Assisted reproduction treatments are very stressful and tiring for infertile couples. Any simplification in the pharmacological treatment is therefore a welcome development [6]. Depot GnRHa in long protocols offers the advantages of better compliance, convenience, and economy for patients, with less stress over injections and risk of infection. However, depot formulations require more gonadotropin ampoules, which are needed for ovarian stimulation, and a significantly longer duration of ovarian stimulation than daily $\mathrm{GnRHa}$ [7].

Another issue related to depot $\mathrm{GnRH}$ agonist is choosing the best dose (half vs. full dose). Yim et al. compared half-dose depot GnRH analogues with fulldose preparations in IVF cycles. They suggested that the use of a lower dose appears to be equally effective and could contribute to a reduction in the cost of treatment [11].

Dal Prato et al. showed that a half dose of depot triptorelin can be successfully used in ovarian stimulation for IVF. In their study, the effect of the different doses of triptorelin (half dose vs. full dose) on the pituitary was quite different. They confirmed that reduced pituitary desensitization is induced by half-dose triptorelin. Furthermore, 1.87 mg triptorelin administration proved sufficient to prevent an $\mathrm{LH}$ surge [6].

Albuquerque et al. in a meta-analysis study [7] showed that there is insufficient evidence that the depot GnRH agonist formulation is better or worse than using a daily $\mathrm{GnRH}$ agonist in the long protocol for pituitary desensitization in IVF cycles; so clinicians should use whichever is more convenient [7]. According to their recommendations, the present study was done on infertile women with both ovaries undergoing their first IVF cycles. In the present study, the efficacy of half-dose, long-acting GnRH agonist (Diphereline) was compared with daily injections of a short-acting GnRH agonist (Suprefact). Although the difference in mean age between the two groups was significant, the mean age of both groups was below 35 years. According to recent work by Gomes, age more than 35 years old, especially 40, had a critical effect on IVF outcome. Gomes et al. divided IVF patients into 3 groups according to their age (age equal to or less than 35 , age $36-39$, and age equal to or more than 40). They found that the number of oocytes obtained in the $\mathrm{G} \geq 40$ group was significantly lower than in the other two groups, but the percentage of good quality embryos was not statistically different and the fertilization rate was similar in the three groups. The average number of transferred embryos and pregnancy rates were statistically lower in the $G$ $\geq 40$ group in comparison with those observed for the $\mathrm{G} \leq 35$ and $36-39$ groups [15].

In the present study, pregnancy rates were comparable in the two groups $(37.1 \%$ in the Diphereline group vs. $37.5 \%$ in the Suprefact group). These findings were in overall agreement with similar studies evaluating half-dose depot $\mathrm{GnRH}$ agonist with daily administration of short-acting forms $[4,16]$.

Taheripanah et al. evaluated the efficacy of lowdose, long-acting GnRHa (Decapeptyl) for pituitary desensitization and compared the outcome of ART cycles to a long protocol of short-acting GnRHa (buserelin). In their report, there was no significant difference in cancellation, pregnancy rate and gonadotropin dose between the two groups [4].

Although the mean numbers of retrieved oocytes, metaphase II oocytes and embryos in the present study were statistically higher in the Diphereline group than in the Suprefact group, because of their significantly lower mean age, we could not draw any conclusion. It could be suggested that the half dose of long-acting $\mathrm{GnRH}$ agonist in comparison to shortacting components did not cause profound pituitary suppression and lead to an adverse effect on ovaries. In addition, use of Diphereline (half dose) is more convenient because of the need for only one injection in comparison with Suprefact, which needs daily injections. The need for a single injection increases patients' compliance for treatment.

In conclusion, this study showed that half-dose, long-acting $\mathrm{GnRH}$ agonist could have a comparable outcome to short-acting components. Also there were no significant adverse effects (including severe OHSS) of long-acting GnRH agonist in comparison to the short-acting analogue. However, further multicentre clinical trials with matched cases and larger sample sizes are recommended.

\section{Acknowledgement}

The authors wish to thank the staff of Royan Institute for their cooperation.

\section{References}

1. Gemzell CA, Kemman E, Jones JR. Premature ovulation during administration of hMG in nonovulatory women. Infertility 1998; 1: 1-10. 
2. Testart J, Lefevre B, Gougeon A. Effects of gonadotrophin releasing hormone agonists $(\mathrm{GnRHa})$ on follicle and oocyte quality. Hum Reprod 1993; 8: 511-8.

3. Tapanainen J, Hovatta O, Juntunen K, et al. Subcutaneous goserelin versus intranasal buserelin for pituitary downregulation in patients undergoing IVF: a randomized comparative study. Hum Reprod 1993; 8: 2052-5.

4. Taheripanah R, Karimzadeh MA, Ghafourzadeh M. Efficacy of Low Dose, Long-acting Gonadotropin Releasing Hormone Analogues (GnRH-a) Compared with Daily Injections of Short-acting GnRH-a in ART Cycles. Iranian Journal of Reproductive Medicine 2004; 2: 29-33.

5. Oyesanya OA, Teo SK, Quah E, Abdurazak N, Lee FY, Cheng WC. Pituitary down-regulation prior to in-vitro fertilization and embryo transfer: a comparison between a single dose of Zoladex depot and multiple daily doses of Suprefact . Hum Reprod 1995; 10: 1042-4.

6. Dal Prato L, Borini A, Coticchio G, Cattoli M, Flamigni C. Half-dose depot triptorelin in pituitary suppression for multiple ovarian stimulation in assisted reproduction technology: a randomized study. Hum Reprod 2004; 19: 2200-5.

7. Albuquerque LE, Saconato H, Maciel MC. Depot versus daily administration of gonadotrophin releasing hormone agonist protocols for pituitary desensitization in assisted reproduction cycles. Cochrane Database Syst Rev 2005; 1.

8. Gonen Y, Dirnfeld M, Goldman S, Koifman M, Abramovici $\mathrm{H}$. The use of long-acting gonadotropin-releasing hormone agonist (GnRH-a; decapeptyl) and gonadotropins versus short-acting $\mathrm{GnRH}-\mathrm{a}$ (buserelin) and gonadotropins before and during ovarian stimulation for in vitro fertilization (IVF). J In Vitro Fert Embryo Transf 1991; 8: 254-9.

9. Cheon KW, Song SJ, Choi BC, et al. Comparison of clinical efficacy between a single administration of long-acting gonadotrophin-releasing hormone agonist (GnRHa) and daily administrations of short-acting $\mathrm{GnRHa}$ in in vitro fertilization-embryo transfer cycles. J Korean Med Sci 2008; 23: 662-6.

10. Balasch J, Gomez F, Casamitjana R, Carmona F, Rivera F, Vanrell JA. Pituitary-ovarian suppression by the standard and half-doses of D-Trp-6-luteinizing hormone-releasing hormone depot. Hum Reprod 1992; 7: 1230-4.

11. Yim SF, Lok IH, Cheung LP, Briton-Jones CM, Chiu TT, Haines CJ. Dose-finding study for the use of long-acting gonadotrophin-releasing hormone analogues prior to ovarian stimulation for IVF. Hum Reprod 2001; 16: 492-4.

12. Tsai HD, Chen CM, Lo HY, Chang CC. Subcutaneous low dose leuprolide acetate depot versus leuprolide acetate for women undergoing ovarian stimulation for in-vitro fertilization. Hum Reprod 1995; 10: 2909-12.

13. Hsieh Y, Tsai H, Chang C, Chang C, Lo H. Comparison of a single half-dose, longacting form of gonadotropinreleasing hormone analog $(\mathrm{GnRH}-\mathrm{a})$ and a short acting form of $\mathrm{GnRH}$-a for pituitary suppression in a controlled ovarian hyperstimulation program. Fertil Steril 2000; 73: 817-20.

14. Conaghan, J, Hardy, K, Handyside AH, Winston R, Leese $H$. Selection criteria for human embryo transfer: a comparison of pyruvate uptake and morphology. J Assist Reprod Genet 1993; 10: 21-30.

15. Gomes LM, Canha Ados S, Dzik A, Novo NF, Juliano Y, Dos Santos SI, Cavagna M.The age as a predictive factor in in vitro fertilization cycles. Rev Bras Ginecol Obstet 2009; 31: 230-4.

16. Safdarian L, Soltani Mohammadi F, Alleyassin A, Aghahosseini M, Alipasha M, Rahimi E. Clinical outcome with half-dose depot triptorelin is the same as reduced- dose daily buserelin in a long protocol of controlled ovarian stimulation for ICSI /embryo transfer: a randomized double-blind clinical trial (NCT00461916). Hum Reprod 2007; 22: 2449-54. 